\title{
OPEN High rate of major drug-drug interactions of lopinavir-ritonavir for COVID-19 treatment
}

\author{
Juan Macías ${ }^{1 凶}$, Ana Pinilla ${ }^{1}$, Francisco A. Lao-Dominguez ${ }^{2}$, Anaïs Corma ${ }^{1}$, \\ Enrique Contreras-Macias ${ }^{2}$, Alejandro González-Serna ${ }^{1}$, Antonio Gutierrez-Pizarraya ${ }^{2}$, \\ Marta Fernández-Fuertes ${ }^{1}$, Ramón Morillo-Verdugo ${ }^{2}$, Marta Trigo ${ }^{1}$, Luis M. Real ${ }^{1}$ \& \\ Juan A. Pineda ${ }^{1}$
}

The impact of drug-drug interactions (DDI) between ritonavir-boosted lopinavir (LPV-r) to treat patients with coronavirus disease 2019 (COVID-19) and commonly used drugs in clinical practice is not well-known. Thus, we evaluated the rate and severity of DDI between LPV-r for COVID-19 treatment and concomitant medications. This was a cross-sectional study including all individuals diagnosed of SARS-CoV-2 infection treated with LPV-r and attended at a single center in Southern Spain (March 1 st to April 30th, 2020). The frequency [ $95 \%$ confidence interval $(95 \% \mathrm{Cl})]$ of potential and major DDI were calculated. Overall, 469 patients were diagnosed of COVID-19, 125 (27\%) of them were prescribed LPV-r. LPV-r had potential DDI with concomitant medications in $97(78 \%, 95 \% \mathrm{Cl} 69-85 \%)$ patients, and in $33(26 \%, 95 \% \mathrm{Cl} 19-35 \%)$ individuals showed major DDI. Twelve (36\%) patients with major DDI and $14(15 \%)$ individuals without major DDI died $(p=0.010)$. After adjustment, only the Charlson index was independently associated with death [adjusted OR $(95 \% \mathrm{Cl})$ for Charlson index $\geq 5$ : 85 (10-731), $p<0.001]$. LPV-r was discontinued due to side effects in $31(25 \%)$ patients. Management by the Infectious Diseases Unit was associated with a lower likelihood of major DDI [adjusted odds ratio $(95 \% \mathrm{Cl})$ : $0.14(0.04-0.53), p=0.003)$. In conclusion, a high frequency of DDI between LPV-r for treating COVID-19 and concomitant medications was found, including major DDI. Patients with major DDI showed worse outcomes, but this association was explained by the older age and comorbidities. Patients managed by the Infectious Diseases Unit had lower risk of major DDI.

Since December 2019, the severe acute respiratory syndrome coronavirus 2 (SARS-CoV-2) infection has become pandemic in a few months. The rapid spread of this novel coronavirus has been further complicated by a wide spectrum of SARS-CoV-2 disease (COVID-19), spanning asymptomatic or mild disease to multiorgan failure $^{1-3}$. Lacking efficacy-proved treatments, the approach to the management of COVID-19 has been largely empirical, based on indirect data on the antiviral activity of compounds not formally assessed in randomized controlled clinical trials for safety and efficacy ${ }^{4,5}$.

Ritonavir-boosted lopinavir (LPV-r) was initially used as antiviral for SARS-CoV-2 infection based on indirect data. Thus, LPV-r has in vitro inhibitory activity against severe acute respiratory syndrome coronavirus (SARS$\mathrm{CoV})^{6}$. In addition, a non-randomized study suggested a positive clinical and virological effect of LPV-r added to ribavirin among patients with SARS-CoV infection ${ }^{7}$. LPV-r showed activity against Middle East respiratory syndrome coronavirus (MERS-CoV) in vitro and in an animal model ${ }^{8,9}$. Viral clearance was reported for some MERS-CoV cases receiving combinations including LPV- $\mathrm{r}^{10}$. Finally, clinical trials randomizing patients with COVID-19 to standard of care or standard of care plus LPV-r has been reported ${ }^{11,12}$. Both trials failed to find differences in the primary objective. In the trial by Cao et al., however, the median time to clinical improvement was shorter in the group treated with LPV-r in the modified intention-to-treat analysis ${ }^{11}$. LPV-r is no longer recommended as treatment for COVID-19.

One issue with LPV-r is the potential for drug-drug interactions (DDI) affecting a wide variety of commonly used medications ${ }^{13}$. Among those, lipid lowering drugs, antiarrhythmics, cardiovascular drugs, analgesics or anticoagulants are frequently used by aged patients. These older patients are more likely to present with more severe COVID-19 requiring admission and respiratory support, and, due to this, more likely candidates to receive

${ }^{1}$ Infectious Diseases and Microbiology Unit, Hospital Universitario Virgen de Valme, Avda Bellavista s/n, 41014 Seville, Spain. ${ }^{2}$ Pharmacy, Hospital Universitario Virgen de Valme, Seville, Spain. ${ }^{\circledR}$ email: juan.macias.sanchez@gmail.com 


\begin{tabular}{|l|c|}
\hline Characteristic & Value \\
\hline Median (Q1-Q3) age, years & $63(53-76)$ \\
\hline Male sex, n (\%) & $60(48)$ \\
\hline Health care professional, n (\%) & $14(11)$ \\
\hline Nursing home residency, n (\%) & $4(3.2)$ \\
\hline Comorbidities*, n (\%) & $25(20)$ \\
\hline Arterial hypertension & $11(8.8)$ \\
\hline Type 2 diabetes mellitus & $18(14)$ \\
\hline Cardiovascular disease & $4(3.2)$ \\
\hline Chronic renal disease & $6(4.8)$ \\
\hline Chronic lung disease & $2(1.6)$ \\
\hline Cirrhosis & $14(11)$ \\
\hline Dementia & $7(5.6)$ \\
\hline Cancer & $3(2.4)$ \\
\hline Drug-related immunosuppression & $1(0.8)$ \\
\hline HIV infection & $15(12)$ \\
\hline Charlson index, n (\%) & $47(38)$ \\
\hline 0 & $37(30)$ \\
\hline $1-2$ & $26(21)$ \\
\hline $3-4$ & $15(12)$ \\
\hline$\geq 5$ & $96(77)$ \\
\hline COVID-19 clinical presentation, n (\%) & $12(9.6)$ \\
\hline Extrapulmonary infection & $2(1.6)$ \\
\hline Pneumonia & $3(2.4)$ \\
\hline Severe pneumonia & \\
\hline Acute severe respiratory distress syndrome & \\
\hline SARS-CoV-2 diagnosis, n (\%) & \\
\hline PCR & \\
\hline Serology & \\
\hline \multicolumn{2}{|l|}{} \\
\hline
\end{tabular}

Table 1. Characteristics of the patients $(n=125)$. ${ }^{a}$ Main comorbidity per patient: for each patient a single comorbidity was selected, i.e. the condition considered most severe.

drugs with potential antiviral activity against SARS-CoV-2, as LPV-r. Management of LPV-r by non-infectious diseases doctors, not used to the DDI profile of this drug, along with the need for a fast response and the overwhelming burden of COVID-19 in some areas might have led to a high rate of major DDI between LPV-r and concomitant prescriptions. Major DDI may even be associated with worse outcomes. However, to our knowledge, there is no data on the frequency and impact of DDI between LPV-r to treat patients with COVID-19 and commonly used drugs in clinical practice.

The pandemic is far from an end, as it is following a relapsing course with new waves of infections. With novel drugs for SARS-CoV-2 becoming available, the issue of DDI with concomitant medications persist. Factors potentially associated with DDI in the context of the COVID-19 health crisis need to be understood to better prevent them. To gain insight into those factors, we evaluated the rate and severity of DDI between LPV-r for COVID-19 treatment and comedications, and the factors associated with those DDI.

\section{Results}

Characteristics of the study population. Four hundred and sixty-nine patients had a confirmed diagnosis of SARS-CoV-2 infection during the study period. Among them, 125 (27\%) individuals were prescribed LPV-r for COVID-19. The median (Q1-Q3) duration of LPV-r treatment was 5 (3-7) days, ranging from 1 to 14 days. Most patients presented comorbidities detected before SARS-CoV-2 infection (Table 1). Thirtyfour (27\%) patients did not show any previous comorbidity. The median (Q1-Q3) Charlson index was 3 (1-4). Approximately one quarter of the study population showed a Charlson index $\geq 5$ (Table 1). The most frequent clinical presentation of LPV-r-treated patients was pneumonia (Table 1). One hundred and twenty-one (97\%) patients were admitted to the hospital, $14(12 \%)$ of them required intensive care, and $26(21 \%)$ died. The characteristics of the study population are summarized in Table 1 .

Frequency and severity drug-drug interactions. Overall, LPV-r had DDI with concomitant medications in $103(82 \%, 95 \%$ CI 75-89\%) subjects. In 97 (78\%, 95\% CI 69-85\%) patients, potential DDI were disclosed, and in 33 (26\%, 95\% CI 19-35\%) individuals major DDI were found. Concomitant medications with potential DDI classified by therapeutic group are summarized in Fig. 1a. Drugs with major DDI with LPV-r are shown in Fig. 1b. Twenty-two (23\%) of 97 individuals undergoing potential DDI between LPV-r and their 


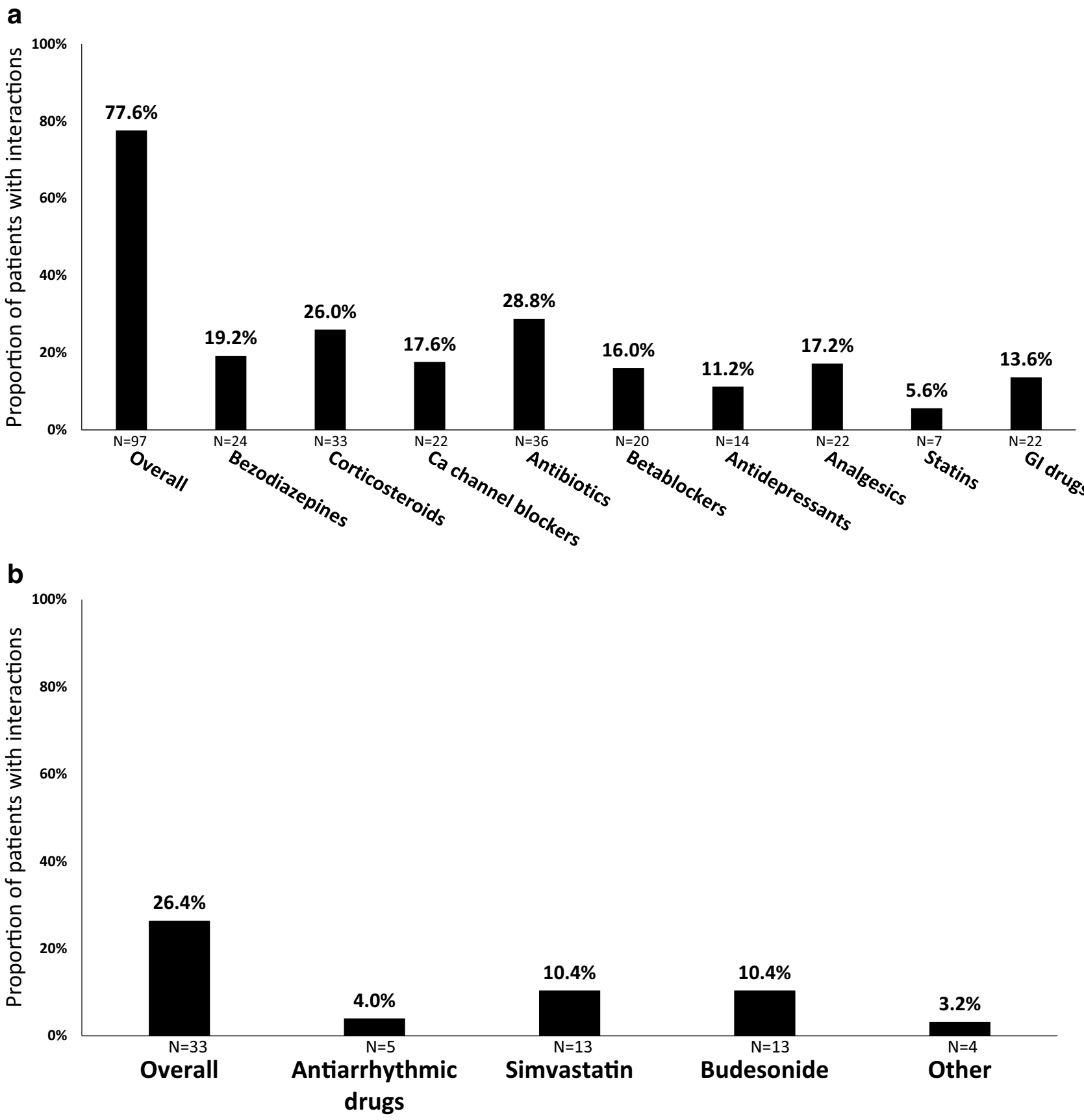

Figure 1. (A) Proportion of patients with potential drug-drug interactions by concomitant medication group $(\mathrm{N}=125)$. GI drugs: gastrointestinal drugs included ondansetron, metoclopramide and loperamide. (B) Proportion of patients with major drug-drug interactions by concomitant medication $(\mathrm{N}=125)$. Other drugs: ivabradine, ticagrelor, domperidone, quetiapine.

concomitant medication died compared with $4(14 \%)$ of 28 patients without potential DDI $(\mathrm{p}=0.335)$. Twelve $(36 \%)$ of 33 patients with major DDI and $14(15 \%)$ of 92 individuals without major DDI died $(\mathrm{p}=0.010)$. After adjustment, only the Charlson index was independently associated with death (Table 2).

Among patients managed in the Infectious Diseases Unit, 41 (76\%) of 54 individuals showed potential DDI compared with $56(79 \%)$ of 71 patients treated outside the Infectious Diseases Unit $(\mathrm{p}=0.695)$. Major DDI were observed in $9(17 \%)$ patients vs. $24(34 \%)$ patients managed by the Infectious Diseases Unit vs. units other than the Infectious Diseases Unit, respectively $(\mathrm{p}=0.031)$. The median Charlson index was $2(1-4)$ compared with $3(2-4)$ for patients managed by the Infectious Diseases Unit and by other units, respectively $(p=0.024)$. The proportion of patients with major DDI treated within the Infectious Diseases Unit by the Charlson index was: Charlson index 0-2, 2 (6.1\%); Charlson index 3-4, 1 (8.3\%); Charlson index $\geq 5,6(67 \%)(\mathrm{p}<0.001)$. The proportion of patients with major DDI managed by other units by the Charlson index was: Charlson index $0-2,7$ (24\%); Charlson index 3-4, $11(44 \%)$; Charlson index $\geq 5,6(35 \%)(p=0.303)$. After adjustment by sex and the 


\begin{tabular}{|c|c|c|c|c|c|c|}
\hline Characteristic & $\mathbf{N}$ & $\begin{array}{l}\text { Deaths } \\
\text { n (\%) }\end{array}$ & $\begin{array}{l}\text { Unadjusted OR (95\% } \\
\text { CI) }\end{array}$ & Univariate $p$ value & Adjusted OR (95\% CI) & Multivariate $p$ value \\
\hline \multicolumn{4}{|l|}{ Age $^{\text {a }}$ (years) } & $<0.001$ & & - \\
\hline$<65$ & 64 & $3(4.7)$ & & & & \\
\hline$\geq 65$ & 61 & $23(38)$ & $12(3.5-44)$ & & & \\
\hline \multicolumn{4}{|l|}{ Sex } & 0.819 & & 0.980 \\
\hline Men & 60 & $13(22)$ & $1.1(0.5-2.6)$ & & $1.0(0.4-2.9)$ & \\
\hline Women & 65 & $13(20)$ & & & & \\
\hline \multicolumn{4}{|l|}{ Charlson index } & $<0.001$ & & $<0.001$ \\
\hline $0-2$ & 62 & $1(1.6)$ & & & Reference & \\
\hline $3-4$ & 37 & $9(24)$ & $19.6(2.4-162)$ & & $18(2.2-151)$ & 0.008 \\
\hline$\geq 5$ & 26 & $16(62)$ & $98(11-820)$ & & $85(10-731)$ & $<0.001$ \\
\hline \multicolumn{4}{|l|}{ Drug-drug interactions } & 0.010 & & 0.383 \\
\hline Not major & 92 & $14(15)$ & $3.2(1.3-7.9)$ & & & \\
\hline Major & 33 & $12(36)$ & & & $1.6(0.6-4.8)$ & \\
\hline \multicolumn{4}{|l|}{ Management } & 0.584 & & - \\
\hline Infectious diseases unit & 54 & $10(19)$ & & & & \\
\hline \begin{tabular}{|l|} 
Other units \\
\end{tabular} & 71 & $16(23)$ & $1.3(0.5-3.1)$ & & & \\
\hline
\end{tabular}

Table 2. Factors associated with death among patients with COVID-19 receiving lopinavir-ritonavir $(n=125)$. a Age was not included in the multivariate analysis because the Charlson index contains this variable.

Charlson index, management by the Infectious Diseases Unit was independently associated with a lower likelihood of major DDI [adjusted odds ratio (95\% CI): $0.14(0.04-0.53), \mathrm{p}=0.003)$.

LPV-r was discontinued due to side effects in 31 (25\%) patients. One patient with pre-existing chronic endstage renal failure suffered grade 3 diarrhea. LPV-r was discontinued, but renal failure worsened, and the patient ultimately died. Another patient with cardiovascular disease was treated with amiodarone for cardiac arrhythmia. He presented an orthostatic syncope, and LPV-r was discontinued, but he died of sudden death afterwards.

\section{Discussion}

In the present study, we found a high overall frequency of DDI between LPV-r for treating COVID-19 and concomitant medications. Moreover, nearly one third of the patients were treated with commonly used medications with major DDI with LPV-r. Patients in whom major DDI were observed showed a worse clinical outcome, but this association was explained by the older age and comorbidities of these patients. Finally, patients managed by the Infectious Diseases Unit had lower risk of major DDI.

Coadministration of LPV-r with drugs that are major substrates of and highly dependent on CYP3A for clearance is associated with increased plasma concentrations of such drugs or their metabolites. In our study, many commonly used medications with that metabolic interaction with LPV-r were prescribed to patients with COVID-19. Antiarrhythmics, symvastatin or budesonide were found to be administered to the study patients. Elevated plasma concentrations of some of those medications are associated with life-threatening reactions. In this regard, we found a significantly higher frequency of deaths among patients who were treated with medications with major DDI with LPV-r. However, after adjustment in a multivariate model, drugs with major DDI with LPV-r were not independently associated with the risk of death. Instead, the main predictor of death was the Charlson index. Therefore, the patient profile, i.e. older individuals with concomitant diseases needing drugs posing contraindications with LPV-r, accounted for the association between deaths and major interactions with LPV-r.

Patients with COVID-19 treated with LPV-r in the present study were severely ill. The majority needed admission and had high Charlson index scores. More importantly, the overall death rate was $21 \%$, i.e. greater than the mortality reported in hospitalized patients in China ${ }^{1,2}$. The older age of patients and their elevated Charlson index could explain the higher death rate in our study.

Discontinuations of LPV-r due to adverse events among HIV-infected patients were lower than $10 \%$ after 2 years of antiretroviral treatment ${ }^{14}$. In a randomized clinical trial of LPV-r for COVID-19 in China, patients stopped early a course of 14 days of LPV-r even more frequently ${ }^{11}$. Nearly $14 \%$ of LPV-r recipients were unable to complete treatment due to adverse events in that trial. In our study, one fourth of the patients discontinued LPV-r because of severe adverse events. The aged and fragile population receiving LPV-r in our study could be more prone to develop LPV-r-related side effects. In fact, our study population was older and accumulated more coexisting conditions than the patients recruited in the Chinese trial of LPV-r for COVID-19 ${ }^{11}$. The elevated frequency of LPV-r discontinuations in fragile patients with severe COVID-19 questions the clinical applicability of LPV-r as antiviral in such patients.

We found that major DDI between comedications and LPV-r were more frequent among patients managed by non-Infectious Diseases Units compared with the Infectious Diseases Unit. After adjustment by the Charlson index, an independent association between major DDI with LPV-r and the treating unit was confirmed. A few factors could explain this finding. First, the overwhelming COVID-19 crisis in our center led to admitting 
patients with COVID-19 in wards other than the Infectious Diseases ward. In this situation, treatment stewardship by Infectious Diseases doctors was not feasible. Second, management by Infectious Diseases specialists has demonstrated to improve the outcome of patients with other infections ${ }^{15,16}$. However, even Infectious Diseases physicians, more aware of the DDI of ritonavir-boosted HIV protease inhibitors than other specialists, exposed patients to major DDI with LPV-r. The complexity of patients, staff shortage due to quarantines of doctors falling ill or exposed to unprotected close contacts, and the many difficulties imposed by a health crisis might partly explain those prescription mistakes.

The present study may have some limitations. First, due to LPV-r supply shortage, this antiviral drug was restricted mostly to more severe patients, those requiring hospitalization. This population represents an extreme of the pandemic, with more complications needing medical intervention and worse outcomes. LPV-r given to younger and less fragile patients might have been more tolerable, with less DDI and discontinuations. Second, post-discharge deaths at home or nursing facilities were not evaluated, only deaths during admissions or at the emergency room were evaluable for the present study. Death rates could have been even higher than those reported herein with a follow-up after discharge, as suggested by another study ${ }^{17}$. Third, the main source of data was the electronic clinical records and the Pharmacy electronic prescriptions. This might have precluded detailed medical record review and it could have led to data loss. However, currently in the Andalusian Health System, health and medical records of every patient are collected in a common electronic database by all caring physicians and nurses. Individual patient electronic medical records were manually evaluated. In addition, all study patients were admitted, and their medications were administered and supervised by hospital nurses. Thus, high adherence to LPV-r and comedications was certain. These are strengths of the present study.

In conclusion, LPV-r for hospitalized patients with COVID-19 frequently interacts with concomitant medications. Major DDI contraindicating the coadministration of LPV-r and common medications were alarmingly overlooked in the setting of the COVID-19 health care crisis. Newer drugs against SARS-CoV-2 infection will need careful studies of DDI. In the wake of the second wave of the pandemic in many countries, DDI of drugs for COVID-19 prescribed by physicians not used to manage them, in patients receiving polymedication for underlying conditions, is a likely issue. Physicians treating patients suffering COVID-19 should be aware of the potential DDI between drugs for COVID-19 and comedications.

\section{Patients and methods}

This was a cross-sectional study. All individuals diagnosed of SARS-CoV-2 infection from March 1st to April 30th, 2020 attended at a single center in Seville, Southern Spain, were evaluated to identify those treated with LPV-r. All patients with COVID-19, confirmed by SARS-CoV-2 PCR or serology, who were prescribed LPV-r as a part of their treatment for SARS-CoV-2 infection were included. The electronic clinical records and Pharmacy electronic prescriptions of all patients were examined to select those who received at least one dose of LPV-r. Concomitant medications were collected from the electronic prescriptions. The electronic clinical records were used to identify comorbid conditions to calculate the Charlson index for each patient ${ }^{13}$. The vital status of patients was established querying electronic clinical records and it was last updated in May 22nd, 2020.

DDI were evaluated using the COVID-19 Drug Interactions site (https://www.covid19-druginteractions.org/) by the Liverpool Drug Interaction Group and the IBM Micromedex Drug Interaction Checking (electronic version, IBM Watson Health, Greenwood Village, Colorado, USA. Available at: https://www.micromedexsolut ions.com/). The frequency and grade of DDI was assessed. DDI were graded, according to the Liverpool Drug Interaction Group, as potential and major. Potential DDI does not preclude co-administration, since they are usually manageable, but they indicate the need to balance risks and benefits for an individual patient. Major DDI corresponds to the "do not co-administer" classification of the Liverpool Drug Interaction Group. After classifying DDI using the COVID-19 Drug Interactions site (https://www.covid19-druginteractions.org/), all DDI were evaluated using the IBM Micromedex Drug Interaction Checking. DDI classified as "contraindicated" or "major" by the IBM Micromedex Drug Interaction Checking were considered as major, and those classified as "moderate" were considered as potential for the present study. Discrepancies in the severity of DDI were resolved classifying DDI as the more severe. The death rate by the severity of DDI was assessed. In addition, side effects leading to discontinuation of LPV-r were examined.

Statistical analysis. Continuous variables were compared by the Student $t$ test, or the Mann-Whitney U if applicable. Categorical variables were compared using the Chi-square test, or the Fisher test where necessary. $95 \%$ confidence intervals ( $95 \% \mathrm{CI}$ ) were calculated for the overall, potential and major frequencies of DDI. Factors associated with death were entered in a logistic regression model, adjusted by age and sex. The STATA 16.0 and IBM SPSS 25 packages were used for the analysis (Supplementary Information S1).

Ethics issues. The study was designed and performed according to the Helsinki declaration and approved by the Ethics Committee of the Valme University Hospital (Seville, Spain). Informed consent was waived by the Ethics Committee of the Valme University Hospital due to the observational retrospective design of the study.

\section{Data availability}

All data generated or analyzed during this study are included in this article.

Received: 23 June 2020; Accepted: 18 November 2020

Published online: 01 December 2020 


\section{References}

1. Chen, N. et al. Epidemiological and clinical characteristics of 99 cases of 2019 novel coronavirus pneumonia in Wuhan, China: A descriptive study. Lancet 395, 507-513 (2020).

2. Huang, C. et al. Clinical features of patients infected with 2019 novel coronavirus in Wuhan, China. Clin. Infect. Dis. 395, 497-506 (2020).

3. Zhang, J. J. Y., Lee, K. S., Ang, L. W., Leo, Y. S. \& Young, B. E. Risk factors of severe disease and efficacy of treatment in patients infected with COVID-19: A systematic review, meta-analysis and meta-regression analysis. Clin. Infect. Dis. https://doi.org/10.1093/ $\mathrm{cid} / \mathrm{ciaa} 576(2020)$.

4. Zhong, H. et al. Efficacy and safety of current therapeutic options for COVID-19-lessons to be learnt from SARS and MERS epidemic: A systematic review and meta-analysis. Pharmacol. Res. 157, 104872 (2020).

5. Fajgenbaum, D. C. et al. Treatments administered to the first 9152 reported cases of COVID-19: A systematic review. Infect. Dis. Ther. https://doi.org/10.1007/s40121-020-00303-8 (2020).

6. Chen, F. et al. In vitro susceptibility of 10 clinical isolates of SARS coronavirus to selected antiviral compounds. J. Clin. Virol. 31, 69-75 (2004).

7. Chu, C. M. et al. Role of lopinavir/ritonavir in the treatment of SARS: Initial virological and clinical findings. Thorax 59, 252-256 (2004).

8. Chan, J.F.-W. et al. Treatment with lopinavir/ritonavir or interferon- $\beta 1 \mathrm{~b}$ improves outcome of MERS-CoV infection in a nonhuman primate model of common marmoset. J. Infect. Dis. 212, 1904-1913 (2015).

9. de Wilde, A. H. et al. Screening of an FDA-approved compound library identifies four small-molecule inhibitors of middle east respiratory syndrome coronavirus replication in cell culture. Antimicrob. Agents Chemother. 58, 4875-4884 (2014).

10. Min, C.-K. et al. Comparative and kinetic analysis of viral shedding and immunological responses in MERS patients representing a broad spectrum of disease severity. Sci. Rep. 6, 25359 (2016)

11. Cao, B. et al. A trial of lopinavir-ritonavir in adults hospitalized with severe covid-19. N. Engl. J. Med. 382, 1787-1799 (2020).

12. Horby, P. W. et al. Lopinavir-ritonavir in patients admitted to hospital with COVID-19 (RECOVERY): A randomised, controlled, open-label, platform trial. Lancet (2020) (Epub ahead of print).

13. Panel on Antiretroviral Guidelines for Adults and Adolescents. Guidelines for the Use of Antiretroviral Agents in Adults and Adolescents with HIV. Department of Health and Human Services.

14. Molina, J.-M. et al. Once-daily atazanavir/ritonavir compared with twice-daily lopinavir/ritonavir, each in combination with tenofovir and emtricitabine, for management of antiretroviral-naive HIV-1-infected patients: 96-week efficacy and safety results of the CASTLE study. J. Acquir. Immune Defic. Syndr. 53, 323-332 (2010).

15. Jenkins, T. C., Price, C. S., Sabel, A. L., Mehler, P. S. \& Burman, W. J. Impact of routine infectious diseases service consultation on the evaluation, management, and outcomes of Staphylococcus aureus bacteremia. Clin. Infect. Dis. 46, 1000-1008 (2008).

16. Jiménez-Aguilar, P. et al. Unsolicited consultation by infectious diseases specialist improves outcomes in patients with bloodstream infection: A prospective cohort study. J. Infect. 77, 503-508 (2018).

17. Rossi, P. G. et al. Survival of hospitalized COVID-19 patients in Northern Italy a population-based cohort study by the ITACOVID19 Network. Medrxiv. https://doi.org/10.1101/2020.05.15.20103119 (2020).

\section{Acknowledgements}

The authors wish to thank the collaboration of the nurse $\mathrm{M}^{\mathrm{a}}$ Victoria Martínez. This study has not been funded. J.A.P. is recipient of an intensification grant from the Instituto de Salud Carlos III (Grant number Programa-I3SNS).

\section{Author contributions}

Study concept and design: J.M., J.A.P., L.M.R., R.M.V. Acquisition of data: A.P., F.L.D., A.C., E.C.M., A.G.S., A.G.P., M.F.F., M.T. Analysis and interpretation of data: J.M., J.A.P., L.M.R. Drafting of the manuscript: J.M., J.A.P., L.M.R., R.M.V. Critical revision of the manuscript: all authors.

\section{Competing interests}

The authors declare no competing interests.

\section{Additional information}

Supplementary information is available for this paper at https://doi.org/10.1038/s41598-020-78029-3.

Correspondence and requests for materials should be addressed to J.M.

Reprints and permissions information is available at www.nature.com/reprints.

Publisher's note Springer Nature remains neutral with regard to jurisdictional claims in published maps and institutional affiliations.

(c) (i) Open Access This article is licensed under a Creative Commons Attribution 4.0 International License, which permits use, sharing, adaptation, distribution and reproduction in any medium or format, as long as you give appropriate credit to the original author(s) and the source, provide a link to the Creative Commons licence, and indicate if changes were made. The images or other third party material in this article are included in the article's Creative Commons licence, unless indicated otherwise in a credit line to the material. If material is not included in the article's Creative Commons licence and your intended use is not permitted by statutory regulation or exceeds the permitted use, you will need to obtain permission directly from the copyright holder. To view a copy of this licence, visit http://creativecommons.org/licenses/by/4.0/.

(c) The Author(s) 2020 